Pacific

Journal of

Mathematics

A ZETA FUNCTION FOR FLIP SYSTEMS

Young-One Kim, Jungseob Lee, and Kyewon K. Park 


\title{
A ZETA FUNCTION FOR FLIP SYSTEMS
}

\author{
Young-One Kim, Jungseob Lee, and Kyewon K. Park
}

In this paper, we investigate dynamical systems with flip maps, which can be regarded as infinite dihedral group actions. We introduce a zeta function for flip systems, and find its basic properties including a product formula. When the underlying $\mathbb{Z}$-action is conjugate to a topological Markov shift, the flip system is represented by a pair of matrices, and its zeta function is expressed explicitly in terms of the representation matrices.

\section{Introduction.}

Let $(X, T)$ be a topological dynamical system, where $X$ is a topological space and $T: X \rightarrow X$ a homeomorphism. A homeomorphism $F: X \rightarrow X$ is called a flip map or simply a flip for $(X, T)$ if

$$
T F=F T^{-1} \text { and } \quad F^{2}=\text { id. }
$$

We call the triplet $(X, T, F)$ a flip system. It is easy to see that if $(X, T, F)$ is a flip system, then $\left(X, T^{m}, T^{n} F\right)$ is also a flip system for any $m, n \in \mathbb{Z}$. Since the infinite dihedral group $D_{\infty}$ is generated by two elements $a$ and $b$ such that

$$
a b=b a^{-1} \quad \text { and } \quad b^{2}=1,
$$

a flip system can be regarded as a $D_{\infty}$-action of homeomorphisms.

Two flip systems $(X, T, F)$ and $\left(X^{\prime}, T^{\prime}, F^{\prime}\right)$ are said to be conjugate if there is a homeomorphism $\Phi: X \rightarrow X^{\prime}$ such that

$$
\Phi T=T^{\prime} \Phi \quad \text { and } \quad \Phi F=F^{\prime} \Phi .
$$

In this case, we write $(X, T, F) \cong\left(X^{\prime}, T^{\prime}, F^{\prime}\right)$, and $\Phi$ is called a conjugacy from $(X, T, F)$ to $\left(X^{\prime}, T^{\prime}, F^{\prime}\right)$. For an arbitrary flip system $(X, T, F), T$ is a conjugacy from $(X, T, F)$ to $\left(X, T, T^{2} F\right)$ and $F$ is a conjugacy from $(X, T, F)$ to $\left(X, T^{-1}, F\right)$.

Since there is a dynamical system $(X, T)$ which is not conjugate to its time reversal $\left(X, T^{-1}\right)$, not every dynamical system has a flip. See [3, p. 104] and also Example 4.1. On the other hand, any topological Markov shift whose transition matrix is symmetric has a natural flip.

It is well-known that measurable $D_{\infty}$-actions are isomorphic if the underlying $\mathbb{Z}$-actions are Bernoulli of the same entropy. In [7] it is shown that 
if the underlying $\mathbb{Z}$-actions are Kolmogorov and isomorphic, there are examples of non-isomorphic $D_{\infty}$-actions. Unlike the measurable case, we can construct infinitely many non-conjugate flips for a full shift in the topological setting. See Example 4.2.

We establish a zeta function for flip systems which is a conjugacy invariant, and give a finite description of the function when the underlying $\mathbb{Z}$-action is conjugate to a topological Markov shift.

The Artin-Mazur zeta function $\zeta_{T}$ for a dynamical system $(X, T)$, found in $[\mathbf{1}]$, is defined by

$$
\zeta_{T}(t)=\exp \left(\sum_{n=1}^{\infty} \frac{p_{n}}{n} t^{n}\right)
$$

where

$$
p_{n}=\left|\left\{x \in X: T^{n} x=x\right\}\right| \quad(n=1,2, \ldots) .
$$

(We assume that the sequence $\left\{\left(p_{n}\right)^{1 / n}\right\}$ is bounded.) The Artin-Mazur zeta function has the product formula

$$
\zeta_{T}(t)=\prod_{\gamma} \frac{1}{1-t^{|\gamma|}},
$$

where the product is taken over all finite orbits $\gamma$ of $T$.

In [5], D. Lind introduced a zeta function for $\mathbb{Z}^{d}$-actions that generalizes the Artin-Mazur zeta function. It is straightforward to extend the notion to the case of general group actions. Let $G$ be a group, $X$ a set and $\alpha$ : $G \times X \rightarrow X$ a $G$-action on $X$. Then the zeta function $\zeta_{\alpha}$ of the action $\alpha$ is defined formally by

$$
\zeta_{\alpha}(t)=\exp \left(\sum_{H} \frac{p_{H}}{|G / H|} t^{|G / H|}\right) .
$$

Here, the sum is taken over all finite-index subgroups $H$ of $G$, that is, subgroups $H$ such that $|G / H|<\infty$, and $p_{H}$ is defined by

$$
p_{H}=|\{x \in X: \forall h \in H \quad \alpha(h, x)=x\}| .
$$

It is easy to see that this zeta function is automorphism-invariant in the following sense: If $\Psi: G \rightarrow G$ is an automorphism and two $G$-actions $\alpha: G \times X \rightarrow X$ and $\widetilde{\alpha}: G \times X \rightarrow X$ satisfy $\widetilde{\alpha}(g, x)=\alpha(\Psi(g), x)$ for all $(g, x) \in G \times X$, then $\zeta_{\alpha}=\zeta_{\widetilde{\alpha}}$.

We define the zeta function $\zeta_{T, F}$ of a flip system $(X, T, F)$ to be the zeta function $\zeta_{\alpha}$ of the $D_{\infty}$-action $\alpha: D_{\infty} \times X \rightarrow X$ that is given by

$$
\alpha(a, x)=T x \quad \text { and } \quad \alpha(b, x)=F x \quad(x \in X),
$$

where $a$ and $b$ are generators of $D_{\infty}$ which satisfy (1.1). Since the zeta function is automorphism-invariant, our definition does not depend on the choice of the generators $a$ and $b$. Moreover, it is clear that this zeta function 
is a conjugacy invariant. There are, however, non-conjugate flip systems with the same zeta function. See Examples 4.3 and 4.4.

In Section 2, we express the zeta function of flip systems in a more tractable form, and establish some of its basic properties including the product formula. In Section 3, we consider the flip systems $(X, T, F)$ such that $(X, T)$ is conjugate to a topological Markov shift. We prove that such a system can be represented by a pair of matrices (Representation Theorem), and express its zeta function in terms of those matrices. Finally, in Section 4, we conclude this paper with some examples.

\section{The zeta function of a flip system.}

Let $(X, T, F)$ be a flip system, and suppose that $D_{\infty}$ is generated by $a$ and $b$ satisfying (1.1). Let $\alpha: D_{\infty} \times X \rightarrow X$ denote the $D_{\infty}$-action defined by (1.5). For a finite-index subgroup $H$ of $D_{\infty}$ set $p_{H}=\mid\{x \in X: \forall h \in$ $H, \alpha(h, x)=x\} \mid$ and suppose that $p_{H}<\infty$ for all finite-index subgroups $H$ of $D_{\infty}$.

In order to express $\zeta_{\alpha}$ explicitly, we need to identify all the finite-index subgroups of $D_{\infty}$. Suppose that $H$ is a finite-index subgroup of $D_{\infty}$. Then there is an integer $k \neq 0$ such that $a^{k} \in H$, since otherwise we must have $|H| \leq 2$. Hence, either $H$ is generated by $a^{i}$ for some integer $i \neq 0$ or by $a^{i}$ and $a^{j} b$ for some integers $i$ and $j$ with $i \neq 0$.

Let $H(i)$ denote the subgroup generated by $a^{i}$, and $H(i, j)$ the one generated by $a^{i}$ and $a^{j} b$. Then it is clear that $H(i)=H(k)$ if and only if $|i|=|k|$, and that $H(i, j)=H(k, l)$ if and only if $|i|=|k|$ and $j-l$ is a multiple of $i$. Moreover, $\left|D_{\infty} / H(i)\right|=2|i|$ and $\left|D_{\infty} / H(i, j)\right|=|i|$ for $i \neq 0$. Therefore we obtain the following:

Lemma 2.1. Let $n$ be a positive integer. If $n$ is odd, then

$$
H(n, 0), H(n, 1), \ldots, H(n, n-1)
$$

are all the subgroups of $D_{\infty}$ with index $n$. In addition to these, there is one more such subgroup $H(n / 2)$ if $n$ is even.

For convenience, we set $p_{i}=p_{H(i)}$ and $p_{i, j}=p_{H(i, j)}$. Then we have

$$
\begin{aligned}
p_{i} & =\left|\left\{x \in X: T^{i} x=x\right\}\right| \quad \text { and } \\
p_{i, j} & =\left|\left\{x \in X: T^{i} x=T^{j} F x=x\right\}\right| .
\end{aligned}
$$

Hence (1.4) and Lemma 2.1 imply that

$$
\zeta_{T, F}(t)=\exp \left(\sum_{n=1}^{\infty} \frac{p_{n}}{2 n} t^{2 n}+\sum_{n=1}^{\infty} \sum_{k=0}^{n-1} \frac{p_{n, k}}{n} t^{n}\right) .
$$

Now, observe that $a H(i, j) a^{-1}=H(i, j+2)$. From this, we see that $p_{i, j}=p_{i, j+2}$. Moreover, it is clear that $p_{i, j}=p_{i, i+j}$. Hence we obtain the 
following:

$$
\sum_{k=0}^{n-1} \frac{p_{n, k}}{n}= \begin{cases}p_{n, 0} & \text { if } n \text { is odd } \\ \left(p_{n, 0}+p_{n, 1}\right) / 2 & \text { if } n \text { is even. }\end{cases}
$$

By (1.2) we have

$$
\exp \left(\sum_{n=1}^{\infty} \frac{p_{n}}{2 n} t^{2 n}\right)=\sqrt{\zeta_{T}\left(t^{2}\right)} .
$$

Theorem 2.2. The zeta function $\zeta_{T, F}$ of the flip system $(X, T, F)$ is given by

$$
\zeta_{T, F}(t)=\sqrt{\zeta_{T}\left(t^{2}\right)} \exp \left(G_{T, F}(t)\right),
$$

where $\zeta_{T}$ is the Artin-Mazur zeta function of $(X, T)$, and

$$
G_{T, F}(t)=\sum_{m=1}^{\infty}\left(p_{2 m-1,0} t^{2 m-1}+\frac{p_{2 m, 0}+p_{2 m, 1}}{2} t^{2 m}\right) .
$$

Proof. The theorem is an immediate consequence of (2.2), (2.3) and (2.4).

Corollary 2.3. Let $R_{T}$ and $R_{T, F}$ denote the radii of convergence of the Maclaurin series of $\zeta_{T}(t)$ and $\zeta_{T, F}(t)$, respectively. If $p_{n}>0$ for some $n$, then we have

$$
0 \leq R_{T} \leq R_{T, F} \leq \sqrt{R_{T}} \leq 1 .
$$

Remark 2.4. If $(X, T)$ is conjugate to a subshift, then it is easy to see that the radius of convergence of $G_{T, F}$ is at least $\exp \left(h_{T} / 2\right)$, where $h_{T}$ is the topological entropy of $(X, T)$. Moreover, if $(X, T)$ is conjugate to a sofic shift, then $h_{T}=\log R_{T}$ (see [6, Chapter 4]), and hence $R_{T, F}=\sqrt{R_{T}}$.

In the remainder of this section, we establish the product formula of the zeta function. Suppose that $\gamma$ is a finite orbit of $(X, T, F)$. Then there is a point $x$ such that $\gamma=\left\{x, T x, \ldots, T^{|\gamma|-1} x\right\}$, or there is a point $x$ such that $\gamma=\left\{x, T x, \ldots, T^{k-1} x\right\} \cup\left\{F x, T F x, \ldots, T^{k-1} F x\right\}$ with $|\gamma|=2 k$. In the first case, we write $\gamma \in \mathcal{O}_{1}$, and in the second case, $\gamma \in \mathcal{O}_{2}$. It is obvious that $\mathcal{O}_{1} \cap \mathcal{O}_{2}=\emptyset$. We denote by $\zeta_{(\gamma)}$ the zeta function of the flip system $\left(\gamma,\left.T\right|_{\gamma},\left.F\right|_{\gamma}\right)$.

Lemma 2.5. If $\gamma \in \mathcal{O}_{1}$,

$$
\zeta_{(\gamma)}(t)=\sqrt{\frac{1}{1-t^{2|\gamma|}}} \exp \left(\frac{t^{|\gamma|}}{1-t^{|\gamma|}}\right),
$$

and if $\gamma \in \mathcal{O}_{2}$,

$$
\zeta_{(\gamma)}(t)=\frac{1}{1-t^{|\gamma|}}
$$


Proof. Let

$$
\begin{aligned}
\widetilde{p}_{i} & =\left|\left\{x \in \gamma: T^{i} x=x\right\}\right| \text { and } \\
\widetilde{p}_{i, j} & =\left|\left\{x \in \gamma: T^{i} x=T^{j} F x=x\right\}\right| .
\end{aligned}
$$

Assume that $\gamma \in \mathcal{O}_{1}$ and $n$ is a positive integer. If $n$ is not a multiple of $|\gamma|$, then no elements of $\gamma$ are fixed by $T^{n}$, and hence $\widetilde{p}_{n}=0$ and $\widetilde{p}_{n, k}=0$ for all $k$. Now suppose $n$ is a multiple of $|\gamma|$. Then every element of $\gamma$ is fixed by $T^{n}$, so that $\widetilde{p}_{n}=|\gamma|$. We can see that if $|\gamma|$ is odd, $\widetilde{p}_{n, 0}=1$; if $|\gamma|$ even, either $\widetilde{p}_{n, 0}=2, \widetilde{p}_{n, 1}=0$ or $\widetilde{p}_{n, 0}=0, \widetilde{p}_{n, 1}=2$. Using (2.2) and (2.3) with $\widetilde{p}_{n}$ and $\widetilde{p}_{n, k}$ in place of $p_{n}$ and $p_{n, k}$ respectively, we have

$$
\zeta_{(\gamma)}(t)=\exp \left(\sum_{m=1}^{\infty} \frac{1}{2 m} t^{2 m|\gamma|}+\sum_{m=1}^{\infty} t^{m|\gamma|}\right)
$$

from which the first assertion follows.

Next, assume that $\gamma \in \mathcal{O}_{2}$. Then for each integer $j$ no elements of $\gamma$ are fixed by $T^{j} F$. Hence $\widetilde{p}_{n, 0}=\widetilde{p}_{n, 1}=0$ for all $n$. Moreover, it is easy to see that $\widetilde{p}_{n}=|\gamma|$ if $n$ is a multiple of $|\gamma| / 2$, and $\widetilde{p}_{n}=0$ otherwise. Again using (2.2) and (2.3) we have

$$
\zeta_{(\gamma)}(t)=\exp \left(\sum_{m=1}^{\infty} \frac{1}{m} t^{m|\gamma|}\right),
$$

from which the second assertion follows.

Theorem 2.6. Let $R_{T, F}$ be the radius of convergence of the Maclaurin series of $\zeta_{T, F}$, and suppose that $R_{T, F}>0$. Then we have

$$
\zeta_{T, F}(t)=\prod_{\gamma \in \mathcal{O}_{1}} \sqrt{\frac{1}{1-t^{2|\gamma|}}} \exp \left(\frac{t^{|\gamma|}}{1-t^{|\gamma|}}\right) \prod_{\gamma \in \mathcal{O}_{2}} \frac{1}{1-t^{|\gamma|}} \quad\left(|t|<R_{T, F}\right) .
$$

Proof. It is clear from the definition that

$$
\zeta_{T, F}(t)=\prod_{\gamma} \zeta_{(\gamma)}(t) \quad\left(|t|<R_{T, F}\right),
$$

where the product is taken over all finite orbits $\gamma$. Now, the result follows from Lemma 2.5.

Let $\mathcal{O}_{T}$ denote the set of all periodic $T$-orbits. It is clear that $\mathcal{O}_{1} \subset \mathcal{O}_{T}$, but a periodic $T$-orbit may not be an orbit of the flip system $(X, T, F)$. We restate Theorem 2.6 as follows:

Theorem 2.7. Let $R_{T, F}$ be the radius of convergence of the Maclaurin series of $\zeta_{T, F}$, and suppose that $R_{T, F}>0$. Then we have

$$
\zeta_{T, F}(t)=\prod_{\beta \in \mathcal{O}_{T}} \sqrt{\frac{1}{1-t^{2|\beta|}}} \prod_{\gamma \in \mathcal{O}_{1}} \exp \left(\frac{t^{|\gamma|}}{1-t^{|\gamma|}}\right) \quad\left(|t|<R_{T, F}\right) .
$$


Proof. Since $\mathcal{O}_{1} \subset \mathcal{O}_{T}$, we have

$$
\prod_{\beta \in \mathcal{O}_{T}} \sqrt{\frac{1}{1-t^{2|\beta|}}}=\prod_{\beta \in \mathcal{O}_{1}} \sqrt{\frac{1}{1-t^{2|\beta|}}} \prod_{\beta \in \mathcal{O}_{T} \backslash \mathcal{O}_{1}} \sqrt{\frac{1}{1-t^{2|\beta|}}} .
$$

Then the right-hand side of (2.5) is equal to

$$
\prod_{\gamma \in \mathcal{O}_{1}} \sqrt{\frac{1}{1-t^{2|\gamma|}}} \exp \left(\frac{t^{|\gamma|}}{1-t^{|\gamma|}}\right) \prod_{\beta \in \mathcal{O}_{T} \backslash \mathcal{O}_{1}} \sqrt{\frac{1}{1-t^{2|\beta|}}} .
$$

In view of Theorem 2.6, we need only to prove the following:

$$
\prod_{\beta \in \mathcal{O}_{T} \backslash \mathcal{O}_{1}} \sqrt{\frac{1}{1-t^{2|\beta|}}}=\prod_{\gamma \in \mathcal{O}_{2}} \frac{1}{1-t^{|\gamma|}} .
$$

We note that if $\beta \in \mathcal{O}_{T} \backslash \mathcal{O}_{1}$, then $F \beta \in \mathcal{O}_{T} \backslash \mathcal{O}_{1}, \beta \cap F \beta=\emptyset$ and $\beta \cup F \beta \in \mathcal{O}_{2}$. Conversely, if $\gamma \in \mathcal{O}_{2}$, then there is an element $\beta_{\gamma} \in \mathcal{O}_{T} \backslash \mathcal{O}_{1}$ such that $\gamma=\beta_{\gamma} \cup F \beta_{\gamma}$. In this case, we have $|\gamma|=2\left|\beta_{\gamma}\right|=2\left|F \beta_{\gamma}\right|$. Thus

$$
\begin{aligned}
\prod_{\beta \in \mathcal{O}_{T} \backslash \mathcal{O}_{1}} \sqrt{\frac{1}{1-t^{2|\beta|}}} & =\prod_{\gamma \in \mathcal{O}_{2}} \sqrt{\frac{1}{1-t^{2\left|\beta_{\gamma}\right|}}} \sqrt{\frac{1}{1-t^{2\left|F \beta_{\gamma}\right|}}} \\
& =\prod_{\gamma \in \mathcal{O}_{2}} \sqrt{\frac{1}{1-t^{|\gamma|}}} \sqrt{\frac{1}{1-t^{|\gamma|}}} \\
& =\prod_{\gamma \in \mathcal{O}_{2}} \frac{1}{1-t^{|\gamma|}} .
\end{aligned}
$$

This proves (2.6).

Corollary 2.8. Let $G_{T, F}$ be as in Theorem 2.2. Then

$$
G_{T, F}(t)=\sum_{\gamma \in \mathcal{O}_{1}} \frac{t^{|\gamma|}}{1-t^{|\gamma|}}
$$

Proof. The result is an immediate consequence of (1.3), Theorem 2.2 and the above theorem.

\section{Flips for topological Markov shifts.}

Let $\mathcal{A}$ be a finite discrete topological space. For $x \in \mathcal{A}^{\mathbb{Z}}$ and $i \in \mathbb{Z}$ the $i$-th coordinate of $x$ is denoted by $x_{i}$, and if $i, j \in \mathbb{Z}$ with $i<j$, the block $x_{i} x_{i+1} \ldots x_{j}$ is denoted by $x_{[i, j]}$. For $x \in \mathcal{A}^{\mathbb{Z}}$, we define $\sigma x$ and $\rho x$ by

$$
(\sigma x)_{i}=x_{i+1} \quad \text { and } \quad(\rho x)_{i}=x_{-i} \quad(i \in \mathbb{Z}) .
$$

Then $\sigma$ and $\rho$ are homeomorphisms of $\mathcal{A}^{\mathbb{Z}}$ onto itself, and satisfy

$$
\sigma \rho=\rho \sigma^{-1} \text { and } \rho^{2}=\mathrm{id},
$$


that is, $\rho$ is a flip for the dynamical system $\left(\mathcal{A}^{\mathbb{Z}}, \sigma\right)$. This dynamical system is called the full $\mathcal{A}$-shift. The map $\sigma$ is called the shift map, and $\rho$ the reverse map. When we express a point as a bi-infinite sequence, we will underline the 0 -th coordinate. For instance, if $x=\ldots x_{-2} x_{-1} \underline{x_{0}} x_{1} x_{2} \ldots$, then $\sigma x=\ldots x_{-2} x_{-1} x_{0} x_{1} x_{2} \ldots$ and $\rho x=\ldots x_{2} x_{1} x_{0} x_{-1} x_{-2} \ldots$

Let $A$ be a $0-1, \mathcal{A} \times \mathcal{A}$ matrix, and $\left(\mathrm{X}_{A}, \sigma_{A}\right)$ denote the topological Markov shift whose transition matrix is $A$. If $A=A^{T}$, then $\mathrm{X}_{A}$ is $\rho$-invariant, and hence $\rho \mid \mathrm{X}_{A}$ is a flip for $\left(\mathrm{X}_{A}, \sigma_{A}\right)$. More generally, if there is a $0-1, \mathcal{A} \times \mathcal{A}$ matrix $P$ such that

$$
A P=P A^{T} \quad \text { and } \quad P^{2}=I,
$$

then there is a flip, denoted by $\phi_{A, P}$, for $\left(\mathbf{X}_{A}, \sigma_{A}\right)$ that is defined as follows: Since $P$ is $0-1$ and $P^{2}=I$, it is a symmetric permutation matrix, that is, $P=P^{T}$ and for each $a \in \mathcal{A}$ there is a unique $a^{*} \in \mathcal{A}$ such that $P\left(a, a^{*}\right)=1$. Then it is easy to see that

$$
\left(a^{*}\right)^{*}=a \quad(a \in \mathcal{A})
$$

and

$$
A(a, b)=1 \Leftrightarrow A\left(b^{*}, a^{*}\right)=1 \quad(a, b \in \mathcal{A}) .
$$

For $x \in \mathrm{X}_{A}$ we define $\phi_{A, P} x$ by

$$
\left(\phi_{A, P} x\right)_{i}=\left(x_{-i}\right)^{*} \quad(i \in \mathbb{Z}) .
$$

Then from (3.2) and (3.3) it follows that $\phi_{A, P}$ is a flip for $\left(\mathbf{X}_{A}, \sigma_{A}\right)$.

The following theorem states that every flip for a topological Markov shift can be represented in this way:

Theorem 3.1 (Representation Theorem). Let $(X, T, F)$ be a flip system, and suppose that $(X, T)$ is conjugate to a topological Markov shift. Then there are 0-1 square matrices $A$ and $P$ satisfying $(3.1)$ such that $(X, T, F)$ is conjugate to $\left(\mathrm{X}_{A}, \sigma_{A}, \phi_{A, P}\right)$.

Proof. We suppose that $(X, T)$ is conjugate to a topological Markov shift $\left(\mathrm{X}_{M}, \sigma_{M}\right)$ through a conjugacy $\Psi$. Set $\phi=\Psi F \Psi^{-1}$. Then this is a flip for $\left(X_{M}, \sigma_{M}\right)$, and $\left(\mathrm{X}_{M}, \sigma_{M}, \phi\right)$ is conjugate to $(X, T, F)$. We will construct a finite set $\mathcal{A}$ and two $0-1, \mathcal{A} \times \mathcal{A}$ matrices $A$ and $P$ satisfying (3.1) such that $\left(\mathrm{X}_{M}, \sigma_{M}, \phi\right) \cong\left(\mathrm{X}_{A}, \sigma_{A}, \phi_{A, P}\right)$.

Since $\phi$ is continuous, there is a positive integer $N$ such that

$$
x_{[-N, N]}=y_{[-N, N]} \Rightarrow(\phi x)_{0}=(\phi y)_{0} \quad\left(x, y \in \mathrm{X}_{M}\right) .
$$

For $x \in \mathrm{X}_{M}$ let $\widetilde{x}$ denote the bi-infinite sequence defined by

$$
\widetilde{x}=\ldots(\phi x)_{2}(\phi x)_{1} \underline{(\phi x)_{0}}(\phi x)_{-1}(\phi x)_{-2} \ldots,
$$

that is, $\widetilde{x}=\rho \phi x$. It should be noted that if $M$ is symmetric, then $\widetilde{x} \in \mathrm{X}_{M}$ for all $x \in \mathrm{X}_{M}$, but in general, this is not the case. For $x \in \mathrm{X}_{M}$ let $[x]$ 
denote the ordered pair of the $(2 N+1)$-blocks $x_{[-N, N]}$ and $\widetilde{x}_{[-N, N]}$, and we express $[x]$ as

$$
[x]=\left[\begin{array}{l}
x_{-N} \ldots x_{0} \ldots x_{N} \\
\widetilde{x}_{-N} \ldots \widetilde{x}_{0} \ldots \widetilde{x}_{N}
\end{array}\right]
$$

Note that if $x, y \in \mathrm{X}_{M}$ and $x_{[-2 N, 2 N]}=y_{[-2 N, 2 N]}$, then $[x]=[y]$.

Now we define $\mathcal{A}$. An ordered pair $\mathbf{a}=\left[\begin{array}{l}a_{-N} \ldots a_{0} \ldots a_{N} \\ \widetilde{a}_{-N} \ldots \widetilde{a}_{0} \ldots \widetilde{a}_{N}\end{array}\right]$ of $(2 N+1)$ blocks is an element of $\mathcal{A}$ if and only if $\mathbf{a}=[x]$ for some $x \in \mathrm{X}_{M}$. It is clear that $\mathcal{A}$ is a finite set. For $\mathbf{a}=\left[\begin{array}{l}a_{-N} \ldots \\ \widetilde{a}_{-N} \ldots a_{0} \ldots a_{0} \ldots a_{N} \\ \widetilde{a}_{N}\end{array}\right] \in \mathcal{A}$ we define

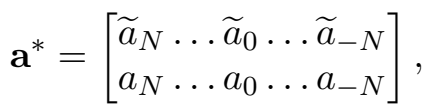

$$
\begin{aligned}
& l(\mathbf{a})=\left[\begin{array}{lllll}
a_{-N} & \ldots & a_{0} & \ldots & a_{N-1} \\
\widetilde{a}_{-N} & \ldots & \widetilde{a}_{0} & \ldots & \widetilde{a}_{N-1}
\end{array}\right], \\
& r(\mathbf{a})=\left[\begin{array}{lllll}
a_{-N+1} & \ldots & a_{0} & \ldots & a_{N} \\
\widetilde{a}_{-N+1} & \ldots & \widetilde{a}_{0} & \ldots & \widetilde{a}_{N}
\end{array}\right], \\
& c(\mathbf{a})=a_{-N} \ldots a_{0} \ldots a_{N} \quad \text { and } \\
& b_{0}(\mathbf{a})=\widetilde{a}_{0} .
\end{aligned}
$$

Obviously $[x]^{*}=[\phi x]$ for all $x \in \mathrm{X}_{M}$. Hence $\mathbf{a}^{*} \in \mathcal{A}$ and $\left(\mathbf{a}^{*}\right)^{*}=\mathbf{a}$ for all $\mathbf{a} \in \mathcal{A}$. Moreover, (3.4) implies that

$$
c(\mathbf{a})=c(\mathbf{b}) \Rightarrow b_{0}(\mathbf{a})=b_{0}(\mathbf{b}) \quad(\mathbf{a}, \mathbf{b} \in \mathcal{A}) .
$$

Next, define the matrices $A$ and $P$ by

$$
A(\mathbf{a}, \mathbf{b})=\delta(r(\mathbf{a}), l(\mathbf{b})) \quad(\mathbf{a}, \mathbf{b} \in \mathcal{A})
$$

and

$$
P(\mathbf{a}, \mathbf{b})=\delta\left(\mathbf{a}^{*}, \mathbf{b}\right) \quad(\mathbf{a}, \mathbf{b} \in \mathcal{A}),
$$

where $\delta$ denotes the Kronecker delta. Then it is straightforward to check that $A$ and $P$ satisfy (3.1).

Finally, define $\Phi: \mathrm{X}_{M} \rightarrow \mathrm{X}_{A}$ by

$$
(\Phi x)_{i}=\left[\left(\sigma_{M}\right)^{i} x\right] \quad\left(x \in \mathrm{X}_{M}, i \in \mathbb{Z}\right) .
$$

Then $\Phi$ is an injective sliding block code of memory and anticipation $2 N$. Moreover a direct calculation shows that $\Phi \phi=\phi_{A, P} \Phi$. It remains only to show that $\Phi$ is surjective. Let $y=\ldots \mathbf{a}_{-2} \mathbf{a}_{-1} \mathbf{a}_{0} \mathbf{a}_{1} \mathbf{a}_{2} \ldots$ be any point in $\mathbf{X}_{A}$. Then there is a point $x \in \mathrm{X}_{M}$ such that

$$
x_{[-N+i, N+i]}=c\left(\mathbf{a}_{i}\right) \quad(i \in \mathbb{Z}) .
$$


Let $z=\Phi x$, and write $z=\ldots \mathbf{b}_{-2} \mathbf{b}_{-1} \mathbf{b}_{0} \mathbf{b}_{1} \mathbf{b}_{2} \ldots$ Then from the definition of $\Phi$, we have

$$
x_{[-N+i, N+i]}=c\left(\mathbf{b}_{i}\right) \quad(i \in \mathbb{Z}) .
$$

Hence $b_{0}\left(\mathbf{a}_{i}\right)=b_{0}\left(\mathbf{b}_{i}\right)$ for all $i \in \mathbb{Z}$ by (3.5), (3.6) and (3.7). This implies $y=z$.

Let $\zeta_{A, P}$ be the zeta function of the flip system $\left(\mathrm{X}_{A}, \sigma_{A}, \phi_{A, P}\right)$. In Theorem 3.2 below, we express $\zeta_{A, P}$ in terms of the matrices $A$ and $P$. It is well-known that the Artin-Mazur zeta function $\zeta_{A}$ of the topological Markov shift $\left(\mathbf{X}_{A}, \sigma_{A}\right)$ satisfies

$$
\zeta_{A}(t)=\frac{1}{\operatorname{det}(I-t A)}
$$

See Theorem 6.4.6 in [6].

We need some notations. For an $\mathcal{A} \times \mathcal{A}$ matrix $B$, the adjugate of $B$ is denoted by $B^{\star}$, so that $B B^{\star}=(\operatorname{det} B) I$, the entry sum $\mathcal{S}[B]$ of $B$ is defined by

$$
\mathcal{S}[B]=\sum_{(a, b) \in \mathcal{A} \times \mathcal{A}} B(a, b),
$$

and the diagonal projection $B^{\Delta}$ of $B$ is defined by

$$
B^{\Delta}(a, b)=B(a, b) \delta(a, b) \quad(a, b \in \mathcal{A}) .
$$

Theorem 3.2. If $A$ and $P$ are 0-1, square matrices which satisfy (3.1), then

$$
\zeta_{A, P}(t)=\sqrt{\zeta_{A}\left(t^{2}\right)} \exp \left(\zeta_{A}\left(t^{2}\right) H_{A, P}(t)\right)
$$

where $H_{A, P}$ is the polynomial defined by

$$
\begin{aligned}
H_{A, P}(t)= & \mathcal{S}\left[t P^{\Delta}\left(I-t^{2} A\right)^{\star}(A P)^{\Delta}\right. \\
& \left.+\frac{t^{2}}{2}\left\{P^{\Delta} A\left(I-t^{2} A\right)^{\star} P^{\Delta}+(P A)^{\Delta}\left(I-t^{2} A\right)^{\star}(A P)^{\Delta}\right\}\right] .
\end{aligned}
$$

Proof. For $i, j \in \mathbb{Z}$ let $p_{i, j}$ denote the number of points in $\mathrm{X}_{A}$ that are fixed by $\left(\sigma_{A}\right)^{i}$ and $\left(\sigma_{A}\right)^{j} \phi_{A, P}$. Set

$$
G_{A, P}(t)=\sum_{m=1}^{\infty}\left(p_{2 m-1,0} t^{2 m-1}+\frac{p_{2 m, 0}+p_{2 m, 1}}{2} t^{2 m}\right) .
$$

Then, in view of Theorem 2.2 and (3.8), we need only to prove the following:

$$
G_{A, P}(t)=\frac{H_{A, P}(t)}{\operatorname{det}\left(I-t^{2} A\right)} .
$$


Let $\mathcal{B}_{n}$ denote the set of all $n$-blocks that occur in points in $\mathrm{X}_{A}$. Then it is easy to see that

$$
\begin{aligned}
p_{2 m+1,0} & =\left|\left\{x_{0} \ldots x_{m} \in \mathcal{B}_{m+1}: x_{0}^{*}=x_{0}, A\left(x_{m}, x_{m}^{*}\right)=1\right\}\right|, \\
p_{2 m, 0} & =\left|\left\{x_{0} \ldots x_{m} \in \mathcal{B}_{m+1}: x_{0}^{*}=x_{0}, x_{m}^{*}=x_{m}\right\}\right|, \quad \text { and } \\
p_{2 m, 1} & =\left|\left\{x_{1} \ldots x_{m} \in \mathcal{B}_{m}: A\left(x_{1}^{*}, x_{1}\right)=A\left(x_{m}, x_{m}^{*}\right)=1\right\}\right| .
\end{aligned}
$$

Recall that for $a \in \mathcal{B}_{1}, a^{*}$ is the unique element of $\mathcal{B}_{1}$ such that $P\left(a, a^{*}\right)=1$. Moreover, for $a, b \in \mathcal{B}_{1}$ the following are obvious:

$$
\begin{aligned}
a^{*}=b & \Leftrightarrow P(a, b)=1, \\
A\left(a, b^{*}\right)=1 & \Leftrightarrow A P(a, b)=1, \quad \text { and } \\
A\left(a^{*}, b\right)=1 & \Leftrightarrow P A(a, b)=1 .
\end{aligned}
$$

Therefore we obtain

$$
\begin{aligned}
p_{2 m+1,0} & =\mathcal{S}\left[P^{\Delta} A^{m}(A P)^{\Delta}\right], \\
p_{2 m, 0} & =\mathcal{S}\left[P^{\Delta} A^{m} P^{\Delta}\right], \quad \text { and } \\
p_{2 m, 1} & =\mathcal{S}\left[(P A)^{\Delta} A^{m-1}(A P)^{\Delta}\right] .
\end{aligned}
$$

On the other hand, we have

$$
\begin{aligned}
& \sum_{m=0}^{\infty} s^{m} A^{m}=(I-s A)^{-1}=\frac{1}{\operatorname{det}(I-s A)}(I-s A)^{\star} \\
& \\
& \quad(s \in \mathbb{C}, \Lambda|s|<1),
\end{aligned}
$$

where $\Lambda$ denotes the spectral radius of $A$. Finally, put (3.11) into (3.9), and use (3.12) to obtain (3.10).

\section{Examples.}

In order for a dynamical system $(X, T)$ to have a flip, it is necessary that $(X, T)$ is conjugate to its time reversal $\left(X, T^{-1}\right)$. However, it is not known whether the condition is sufficient. The first example shows that there is a dynamical system with no flips.

Example 4.1. Let

$$
A=\left[\begin{array}{cc}
19 & 5 \\
4 & 1
\end{array}\right]
$$

and $\left(\mathrm{X}_{A}, \sigma_{A}\right)$ denote the edge shift of $A$. It is known that $A$ is not shift equivalent to its transpose $A^{T}\left[\mathbf{3}, \mathrm{p}\right.$. 104]. Hence $\left(\mathrm{X}_{A}, \sigma_{A}\right)$ is not conjugate to its time reversal $\left(\mathrm{X}_{A}, \sigma_{A}^{-1}\right) \cong\left(\mathrm{X}_{A^{T}}, \sigma_{A^{T}}\right)$. Consequently, $\left(\mathrm{X}_{A}, \sigma_{A}\right)$ does not admit a flip. 
In the remainder of this section, we consider various flips on full shifts. We show that some of them are not conjugate by calculating their zeta functions or counting the number of fixed points.

Example 4.2. Let $(X, \sigma)$ be the full 2-shift. We will show that there are infinitely many non-conjugate flips for $(X, \sigma)$. For each positive integer $n$ we define the $(2 n+5)$-block map $K_{n}$ by $K_{n}\left(110^{2 n+1} 11\right)=1, K_{n}\left(110^{n} 10^{n} 11\right)=$ 0 , and $K_{n}\left(x_{-n-2} \ldots x_{0} \ldots x_{n+2}\right)=x_{0}$ when the block is not equal to any of the above two. Let $\kappa_{n}$ denote the sliding block code on $X$ induced by the block map $K_{n}$. Then clearly $\kappa_{n}$ is an automorphism of order 2. Let $\omega_{n}=\rho \kappa_{n}$, where $\rho$ is the reverse map. It is easy to see that $\omega_{n}$ is a flip map for $(X, \sigma)$. The flip systems $\left(X, \sigma, \omega_{n}\right), n \geq 1$, are not conjugate to each other. In fact, for $1 \leq n<m$,

$$
\left|\left\{x \in X: \sigma^{2 m+5} x=x, \omega_{n} x=x\right\}\right|=2^{m+3}-2^{m-n+1},
$$

and

$$
\left|\left\{x \in X: \sigma^{2 m+5} x=x, \omega_{m} x=x\right\}\right|=2^{m+3}-2 .
$$

From this and Theorem 2.2, it also follows that $\zeta_{\sigma, \omega_{n}}, n \geq 1$, are all distinct. A long but straightforward calculation using Theorem 3.2 yields that the zeta function for $\left(X, \sigma, \omega_{1}\right)$ is equal to

$$
\sqrt{\frac{1}{1-2 t^{2}}} \exp \left(\frac{2 t+3 t^{2}-2 t^{5}-2 t^{6}+2 t^{7}+2 t^{10}-2 t^{12}-2 t^{14}}{1-2 t^{2}}\right) \text {. }
$$

Example 4.3. Let $n \geq 2$ be an integer, $(X, \sigma)$ the full $n$-shift, and $\rho: X \rightarrow$ $X$ the reverse map. As the zeta function is automorphism-invariant, the flip systems $(X, \sigma, \rho)$ and $(X, \sigma, \sigma \rho)$ have the same zeta function, which is

$$
\zeta_{\sigma, \rho}(t)=\sqrt{\frac{1}{1-n t^{2}}} \exp \left(\frac{n t+\left(n+n^{2}\right) t^{2} / 2}{1-n t^{2}}\right) .
$$

They are, however, not conjugate. In fact, we have

$$
\left|\left\{x \in X: \sigma^{2} x=x, \rho x=x\right\}\right|=n^{2},
$$

whereas

$$
\left|\left\{x \in X: \sigma^{2} x=x, \sigma \rho x=x\right\}\right|=n .
$$

As we have seen in the above examples, a dynamical system may have many non-conjugate flip maps. However the following question still remains to be answered: Let $A$ and $B$ be symmetric $0-1$ matrices such that $\left(\mathrm{X}_{A}, \sigma_{A}\right) \cong\left(\mathrm{X}_{B}, \sigma_{B}\right)$. Does it follow that $\left(\mathrm{X}_{A}, \sigma_{A}, \rho_{A}\right) \cong\left(\mathrm{X}_{B}, \sigma_{B}, \rho_{B}\right)$ ?

Example 4.4. Let $(X, \sigma)$ be the full 2-shift, and $\psi: X \rightarrow X$ defined by

$$
\psi(x)=\ldots x_{2}^{*} x_{1}^{*} \underline{x_{0}^{*}} x_{-1}^{*} x_{-2}^{*} \ldots,
$$


where $0^{*}=1$ and $1^{*}=0$. Then $\psi$ is a flip for $(X, \sigma)$. The flips $\psi$ and $\sigma \psi$ are not conjugate since $\psi$ has no fixed points but $\sigma \psi$ has fixed points. But they have the same zeta function

$$
\zeta_{\sigma, \psi}(t)=\sqrt{\frac{1}{1-2 t^{2}}} \exp \left(\frac{t^{2}}{1-2 t^{2}}\right)
$$

On taking $n=2$ in Example 4.3, we know that $\rho$ and $\sigma \rho$ are not conjugate, and have the same zeta function

$$
\zeta_{\sigma, \rho}(t)=\sqrt{\frac{1}{1-2 t^{2}}} \exp \left(\frac{2 t+3 t^{2}}{1-2 t^{2}}\right) .
$$

Therefore the four flips $\rho, \sigma \rho, \psi$ and $\sigma \psi$ for $(X, \sigma)$ are not conjugate to each other.

Example 4.5. Let $\mathcal{A}=\{0,1,2,3\}$. Let $A$ and $P$ be $0-1, \mathcal{A} \times \mathcal{A}$ matrices defined by $A(i, j)=1$ for all $(i, j)$, and $P(i, j)=1$ if and only if $(i, j) \in$ $\{(0,0),(1,1),(2,3),(3,2)\}$. Then we find that the flip system $\left(\mathbf{X}_{A}, \sigma, \phi_{A, P}\right)$ has the zeta function

$$
\zeta_{A, P}=\sqrt{\frac{1}{1-4 t^{2}}} \exp \left(\frac{2 t+4 t^{2}}{1-4 t^{2}}\right) .
$$

Now, we will show that the flips $\phi_{A, P}$ and $\sigma_{A} \phi_{A, P}$ for the full 4-shift $\left(\mathrm{X}_{A}, \sigma_{A}\right)$ are conjugate. Let $X=\{0,1\}^{\mathbb{Z}}, \sigma: X \rightarrow X$ the shift map, and $\rho: X \rightarrow X$ the reverse map. Let $\pi_{1}:\{0,1\}^{2} \ni a b \mapsto a \in\{0,1\}$, and $\pi_{2}:\{0,1\}^{2} \ni a b \mapsto b \in\{0,1\}$. Define $f: \mathcal{A} \rightarrow\{0,1\}^{2}$ by $f(0)=00$, $f(1)=11, f(2)=01$ and $f(3)=10$, and $\Phi: \mathrm{X}_{A} \rightarrow X$ by

$$
\Phi(x)=\ldots \pi_{1} f\left(x_{-1}\right) \pi_{2} f\left(x_{-1}\right) \pi_{1} f\left(x_{0}\right) \underline{\pi_{2} f\left(x_{0}\right)} \pi_{1} f\left(x_{1}\right) \pi_{2} f\left(x_{1}\right) \ldots
$$

We can easily check that $\Phi$ is a conjugacy from $\left(\mathbf{X}_{A}, \sigma_{A}, \phi_{A, P}\right)$ to $\left(X, \sigma^{2}, \sigma \rho\right)$, and so one from $\left(\mathbf{X}_{A}, \sigma_{A}, \sigma_{A} \phi_{A, P}\right)$ to $\left(X, \sigma^{2}, \sigma^{3} \rho\right)$. Trivially $\sigma$ is a conjugacy from $\left(X, \sigma^{2}, \sigma \rho\right)$ to $\left(X, \sigma^{2}, \sigma^{3} \rho\right)$. Therefore $\Phi^{-1} \sigma \Phi$ is a conjugacy from $\left(\mathrm{X}_{A}, \sigma_{A}, \phi_{A, P}\right)$ to $\left(\mathbf{X}_{A}, \sigma_{A}, \sigma_{A} \phi_{A, P}\right)$. This proves the assertion.

Acknowledgment. The authors would like to thank Professors R. Burton, K. H. Kim and F. Roush for their helpful comments in the preparation of this paper.

\section{References}

[1] M. Artin and B. Mazur, On periodic points, Ann. of Math., 81 (1965), 82-99, MR 31 \#754, Zbl 0127.13401.

[2] R. Bowen and O. Lanford, Zeta functions of restrictions of the shift transformation, Proc. Sympos. Pure Math., 14 (1970), 43-49, MR 42 \#6284, Zbl 0211.56501. 
[3] M. Boyle, B. Marcus and P. Trow, Resolving maps and the dimension group for shifts of finite type, Mem. Amer. Math. Soc., 377 (1987), MR 89c:28019, Zbl 0651.54018.

[4] R. Burton, Private communications.

[5] D. Lind, A zeta function for $\mathbb{Z}^{d}$-actions, London Math. Soc. Lecture Note Ser., 228 (M. Pollicott and K. Schmidt, ed.), 1996, 433-450, MR 97e:58185, Zbl 0881.58052.

[6] D. Lind and B. Marcus, An Introduction to Symbolic Dynamics and Coding, Cambridge University Press, 1995, MR 97a:58050.

[7] K.K. Park, On ergodic foliations, Ergodic Theory Dynam. Systems, 8 (1988), 437-457, MR 90b:28021, Zbl 0627.28015.

Received December 26, 2001. This research was supported by the Brain Korea 21 Project.

School of Mathematical Sciences

Seoul National University

SEOUL 151-747

KorEA

E-mail address: kimyo@math.snu.ac.kr

Department of Mathematics

AJou University

SUWON 442-749

KoreA

E-mail address: jslee@madang.ajou.ac.kr

Department of Mathematics

AJou University

SUWON 442-749

KoreA

E-mail address: kkpark@madang.ajou.ac.kr 